\title{
REFLEXIONES SOBRE CULTURA, EDUCACIÓN, DEPORTE Y ENTRETENIMIENTO BAJO LA ÓPTICA DE LA EDUCACIÓN FÍSICA BRASILEÑA ${ }^{1}$
}

\author{
REFLECTIONS ON CULTURE, EDUCATION, SPORT AND ENTERTAINMENT IN THE OPTICS OF \\ PHYSICAL EDUCATION IN BRAZIL
}

Lino Castellani Filho ${ }^{2}$

\section{Resumen}

\begin{abstract}
En épocas de megaeventos deportivos en suelo brasileño, donde más que nunca el deporte como negocio/ mercadería se pone en evidencia al costo de la disminución de su comprensión como práctica social constituyente del patrimonio cultural de un pueblo, y el tiempo libre se transforma en tiempo y espacio de consumo bajo la lógica de la industria cultural del entretenimiento. En este artículo se reflexiona sobre cómo, parte contrahegemónica de la reciente (poco más de 30 años) área académica denominada educación física, se está apropiando de estas prácticas sociales. Ya sea para efecto de estudios, investigaciones y formación profesional/académica, sea para la configuración de políticas públicas educacionales, deportivas y de ocio que se contrapongan a los intereses de los sectores conservadores del sector deportivo, consciente de que lo que aquí pasa refleja -guardadas las especificidades de cada país- el proceso impuesto a los países sudamericanos sometidos a los impactos de la globalización emprendida bajo la lógica neoliberal.
\end{abstract}

Palabras claves: Brasil, educación física, deporte, tiempo libre, políticas deportivas y de tiempo libre.

Abstract

In times of mega sporting events on Brazilian soil where more than ever Sport as a business / merchandise evidenced at the cost of decreasing your understanding as constitutive social practice of cultural heritage of a people, and Free Time becomes time and space consumption in the logic of cultural entertainment. This article is provided to reflect on how, hegemonic party against the recent academic sectors us called Physical Education, is appropriating these social practices. Be for purposes of study, research and professional / academic training, which is for setting up educational, sports and entertainment public policy that are against the interests of the conservative sectors of the sports industry, aware that what is happening here reflects - conserve the specificities of each country-the process imposed on the South American countries under the impact of globalization undertaken under the neoliberal logic.

Keywords: Brazil, physical education, sports, leisure, sports and leisure policy.

Fecha de recepción: 16 de octubre de 2014

Fecha de aprobación: 21 de marzo de 2015

Para citar este artículo:

Castellani Filho, L. (2015). Reflexiones sobre cultura, educación, deporte y entretenimiento

bajo la óptica de la educación física brasileña. Lúdica Pedagógica, (21), 63-75.

1 El presente artículo refleja la actualización y la adaptación al escenario latinoamericano de otro artículo publicado en edición restricta y no comercial bajo la forma de capítulo del libro organizado por Ari Lazzarotti Filho y Fernando Mascarenhas, titulado "Tiempo libre, cultura y educación: contribuciones al debate contemporáneo", publicado en 2010 por la Editorial de la Universidad Federal de Goiás.

2 Doctor en Educación (Unicamp); profesor libre-docente de la Facultad de Educación Física de la Universidad Estatal de Campinas, Unicamp (1986/2011); profesor-visitante de la Universidad Federal de Brasilia (UNB) (2012/13); coordinador del Observatorio del Deporte, Observatorio de Políticas de Educación Física, Deporte y Entretenimiento (CNPQ/Unicamp); secretario Nacional de la Secretaría Nacional de Desarrollo del Deporte y del Tiempo Libre del Ministerio del Deporte (2003/2006); presidente del Colegio Brasileño de Ciencias del Deporte (CBCE) (1999/2001; 2001/2003); presidente de la Asociación de Docentes de la Unicamp, Adunicamp (1996/1998). Correo electrónico: lino.castellani@uol.com.br 


\section{PARA INICIAR LA CONVERSACIÓN}

La previsión de crecimiento de la industria del entretenimiento era de 6,4 \% al año, en promedio, hasta 2011, cuando sus ingresos pasarían de los US\$ 2 billones ( 2 trillones en moneda brasileña). Esto revelaba un estudio de la consultoría Pricewaterhouse Coopers (www. pwc.com). Su principal avance ocurriría en los países del BRIC (Brasil, Rusia, India y China). Es lo que se extraía de su documento Global Entertainment and Media Outlook: 2007-2011. El estudio realiza proyecciones para 14 segmentos de esta industria, como los mercados de televisión, Internet, música, cine, games, libros, revistas y periódicos. Dividido en regiones (EE. UU., EMEA [Europa, Medio Oriente y África], este de Asia, Canadá y Latinoamérica) y abarcando 58 países, prevé para Latinoamérica un crecimiento anual de la industria del entretenimiento del orden de 8,9\% al año.

\section{LA CULTURA GLOBAL ${ }^{3}$}

No hay sociedad, solamente individuos. Margaret Thatcher.

Hobsbawm (1997) imputa al desmoronamiento de la familia tradicional, alcanzada por el nuevo individualismo moral de fines del siglo XX -que tiene en la frase de la ex primera ministra inglesa, por él citada, su expresión mayor- el núcleo básico de la brecha que se abrió entre las reglas de vida y moralidad y la realidad del comportamiento de final de siglo. Conforme Hobsbawn (1997):

Las consecuencias materiales del aflojamiento de los lazos de familia tradicionales fueron talvez aún más serias, ya que [...] la familia no era apenas lo que siempre fuera, un mecanismo para reproducirse, sino también un mecanismo para la cooperación social, [y] como tal, fue esencial para el mantenimiento tanto de la economía agraria como de las primeras economías locales y globales (p. 332).

Una vez que tal institución y sus valores intrínsecos pasaron a no pertenecer más a una perspectiva de ordenamiento social, que relacionaba a las personas entre sí, asegurando la cooperación social y la reproducción

3 El texto siguiente es un extracto del capítulo II (En Tiempos de Globalización) de mi tesis de Doctorado "La Educación Física en el Sistema Educacional Brasileño: Camino, paradojos y perspectivas".
[...] desapareció la mayor parte de su capacidad de estructurar la vida social humana [que] encontró expresión ideológica en una variedad de teorías, del extremo liberalismo de mercado al "posmodernismo" y cosa y tal, que intentaban contornar enteramente el problema de juicio y valores, o antes, reducirlos al único denominador de la irrestricta libertad del individuo (Hobsbawm, 1997, p. 332).

Para Octavio Ianni (1997),

[...] está en marcha la racionalización del mundo, comprendiendo las relaciones, procesos y estructuras con que se perfeccionan la dominación y la apropiación, la integración y el antagonismo [pues] la cultura del capitalismo seculariza todo lo que encuentra por delante y puede transformar muchas cosas en mercancía, inclusive signos, símbolos, emblemas, fetiches [ya que] esta es una exigencia de la racionalización formal, pragmática, definida en términos de fines y medios objetivos, inmediatos [...] cada vez más vacía de valores generales y particulares que no pueden traducirse en los términos del statu quo [es decir], poco a poco, en todos los lugares, regiones, países, continentes, aunque de las diferencias socioculturales que les son propias, los individuos y las colectividades son movidos por la mercancía, mercado, dinero, capital, productividad, lucro (pp. 71-72).

Tal entendimiento se inscribe en una de las dos comprensiones de la cultura global traducidas por Marcos Augusto Gonçalves (1997). En dicha nota, el autor la describe como:

“[...] [la] visión de un mundo crecientemente limpio, informatizado, en el cual los pueblos y los individuos se benefician de las maravillas de la técnica y cultivan la semilla de la conciencia planetaria que triunfará en la aldea global del tercer milenio", asimismo presenta otra noción de cultura global reconocida como el "resultado de la extensión de una determinada cultura a los límites del globo (donde), un mismo sistema de creencias, comportamientos y representaciones se expande sobre la Tierra, suplanta las fronteras nacionales, subyuga la heterogeneidad y se impone como totalidad uniformizada (Gonçalves, 2007, p. 10).

Por lo menos es así que percibimos a Ianni (1997) cuando dice:

[...] bajo varios aspectos, el nuevo ciclo de occidentalización recoloca el problema de la mundialización de la industria cultural, con la expansión de los medios de comunicación de masas y la producción de una cultura de tipo internacional popular 
[donde] se verifica la movilización de todos los recursos disponibles de los medios de comunicación, de los medios en general, impresos y electrónicos, de modo a "reeducar" pueblos, naciones y continentes (p. 73).

Pues el centro irradiador de todo esto, ventilado por Gonçalves, tiene un nombre: imperialismo capitalista, "cuya hegemonía económica, tecnológica y cultural podría ser coronada con la conquista final del planeta" (Gonçalves, 1997, p. 10). Ni una cosa ni otra, con equilibrio, relativiza Gonçalves (1997) al afirmar:

[...] realmente, ninguna mirada podrá aprehender las transformaciones por las que pasa el mundo sin ver el papel desempeñado por la informática, por la robótica, por las comunicaciones por satélite, por la Internet y por los modernos medios de transporte [como también] de la misma forma, es cierto que los estadounidenses dominan la industria cultural en escala mundial y venden su cultura y sus productos a todos los rincones del mundo. Algunos hechos, sin embargo, conspiran tanto contra el fetiche y la apología de la técnica como el determinismo militante (p. 10).

Apuntando los deslices de una y de otra vertiente de la euforia en torno de los medios de comunicación e informática, análoga a la actitud del hombre del siglo XIX frente a la ciencia/tecnología, a aquella de la inexorabilidad de la macdonaldización del planeta, Gonçalves entiende que es natural que en este mundo transformado por la internacionalización "aparezca la nostalgia de la comunidad integrada, que inmoviliza al individuo en un espacio físico, afectivo y simbólico determinado (donde) las relaciones sociales se basan en el cara a cara y donde florecen formas culturales 'verdaderas" (Gonçalves, 1997, p.10). Pues las dificultades de la occidentalización se establecen en el hecho de que

[...] las naciones dominantes y las organizaciones multinacionales actúan de modo diverso, divergente o incluso contradictorio, unas en relación a las otras, y porque los pueblos, grupos, clases, nacionalidades o sociedades no occidentales [...] también poseen su cultura, continúan a producir culturalmente, devuelven elementos culturales occidentales con ingredientes nativos, cuando no lanzan en la sociedad mundial sus producciones originales (Ianni, 1997, pp. 74-75).

Es lo que también nos expone Hobsbawm (1997) en relación a los blue jeans y al rock, al tratar el internacionalismo sin malestares nacionalistas de la nueva cultura joven en las sociedades urbanas:

Esto reflejaba la abrumadora hegemonía cultural de los EE.UU. en la cultura popular y en los estilos de vida, aunque se deba notar que los propios núcleos de la cultura joven occidental eran lo opuesto del chauvinismo cultural, sobre todo en sus gustos musicales. Acogían estilos importados del Caribe, de América Latina y, a partir de la década de 1980, cada vez más, de África (p. 320).

Lo que parece estar evidente en lo expuesto anteriormente es que la mundialización del capital requiere un proceso análogo en la cultura, porque la pluralidad de estándares de vida, de objetos y hábitos de consumo obstaculiza su expansión.

La exigencia de aumentar el número de compradores de objetos proyectados y producidos en forma "standart", requiere la eliminación de diferencias de comportamiento y de gusto dentro de cada nación (entre la ciudad y el campo, entre clases sociales) y también entre países desarrollados y dependientes (García Canclini, 1983, p. 44).

El investigador estudió ya en la primera mitad de la década del ochenta, mucho antes, por lo tanto, de la popularización del fenómeno de la globalización. Conforme el autor, la unificación internacional de los programas ideológicos destinados a la construcción del consenso de los sistemas políticos de control y represión acompaña la homogenización de la economía.

Cada Estado -afirma- uniformiza y centraliza la vida interna de la nación y, al mismo tiempo, coordina el propio sistema o simplemente lo somete a la organización transnacional de la economía y de la cultura [pues] el crecimiento transnacional del capitalismo requiere, al mismo tiempo, la unificación de cada sistema nacional y su subordinación al orden mayor encabezada por las metrópolis (García Canclini, 1983, p. 44).

\section{EL DEPORTE}

El concepto actual de deporte -construcción humana históricamente creada y socialmente desarrollada-, tuvo su elaboración teórico práctica de diferentes formas, todas fruto de convenciones edificadas y difundidas a lo largo de la historia. Lo que más se aproxima de una posición consensualmente aceptada o entendida, en las palabras de Valter Bracht (1997) como 
[...] una práctica social situada en el cuadro de la cultura corporal surgida en el ámbito de la cultura europea, alrededor del siglo XVIII, con carácter competitivo, cuya ejecución reposa esencialmente sobre la idea de lucha contra un elemento definido: una distancia, una duración, un obstáculo, una dificultad material, un peligro, un adversario (s.p.) ${ }^{4}$.

Aunque el estudio citado en el inicio no haga mención al deporte, ya se ve integrado a los temas absorbidos por la industria del entretenimiento, que lo tiene como mercancía altamente rentable y, por tanto, cada vez más distante del concepto de riqueza inmaterial inherente al universo de la cultura, a ser apropiada por todos, y sí cada vez más vinculado al entendimiento de la ciudadanía confundida con capacidad de consumir, donde el par ciudadano/consumidor se impone olímpicamente.

Se sabe que es responsable por un considerable porcentaje del producto interno bruto (PIB) brasileño $0^{5}$. Su cadena productiva es envidiable, y si desean saber más del asunto, no busquen en las páginas deportivas, sino en las de los suplementos de negocios ${ }^{6}$. Los Juegos Panamericanos realizados en 2007 en Río de Janeiro fueron difundidos por los medios de comunicación y pudieron ser acompañados por todos los que lo deseaban. Gran parte de las noticias divulgadas apareció en las columnas políticas y económicas y los acuerdos para la realización del Campeonato Mundial de Fútbol en 2014, y las Olimpiadas en 2016 siguen los mismos caminos, pudiendo también estar presentes en la columna policial, donde relatos sobre transacciones de grandes (!) clubes brasileños se hacen cada día más frecuentes ${ }^{7}$.

4 El documento Política Nacional del Deporte, aprobado por el Consejo Nacional del Deporte en Junio de 2005, así como el Programa Deporte y Tiempo libre de la Ciudad (PPA 2004/07), de los cuales participamos en su elaboración en la condición de Secretario Nacional de la SNDEL/ME - Secretaría Nacional de Desarrollo del Deporte y del Tiempo libre del Ministerio del Deporte -, incorpora la comprensión aquí reproducida.

5 Datos del Ministerio del Deporte de 2004 indicaban algo alrededor del $2 \%$. En 2006 el porcentaje mencionado ya estaba próximo al $4 \%$.

6La revista Carta Capital publica una columna, firmada por Fabio Kadow, denominada "Deporte $S / A$ ". Es encontrada en la sección "Plus-Valía". ¡No podría ser más sutil la crítica presente! ¿O no es crítica?

7 Es sabido que la naturaleza jurídica de derecho privado de las entidades de administración y práctica deportiva, es responsable por un cuadro de autonomía impeditivo de intervención gubernamental, lo que no sería problema si no fuera la total incapacidad de sobrevivir sin subsidios públicos. El actual Gobierno, por la manera como estructuró la instancia gubernamental responsable

\section{EL TIEMPO LIBRE}

Fenómeno típicamente moderno resultante de las tensiones entre capital y trabajo, que se materializa como un tiempo y espacio de vivencias lúdicas, lugar de organización de la cultura, postergado por relaciones de hegemonía. Es así que uno de sus estudiosos. (Mascarenhas, 2005, s.p.) ${ }^{8}$

Típica institución de la sociedad industrial, nacida con la escondida intención de ampliar el control social de los dueños de los medios de producción sobre los trabajadores, para más allá del tiempo de trabajo de ellos, el tiempo libre se configuró a partir de una fuerte tendencia funcionalista, colocándose a servicio de la reproducción de los hábitos, valores y sentidos necesarios para el mantenimiento del modo de producción vigente $^{9}$.

El fortalecimiento de la lógica propia a la sociedad de consumo hizo, por otro lado a su vez, estructurar el tiempo/espacio de tiempo libre a través de los referenciales de interés de la industria del entretenimiento, construyendo las vías de acceso a partir de criterios centrados en el nivel socioeconómico de la población de las sociedades modernas.

El cuadro revelado en nuestro país, a partir de esta situación, favorece la constatación de una (solamente) aparente paradoja. En él se detecta un aumento del consumo por parte de los brasileños, de aquellos artículos asociados al universo del entretenimiento ${ }^{10}$, al

por la definición de la política deportiva (Ministerio del Deporte) se transformó en rehén de los sectores conservadores que hegemonizan el sector deportivo -aquí comprendido en la lógica establecida por Bourdieu- frustrando las expectativas de los que luchaban y luchan por la reversión de esta correlación de fuerzas.

8 Fernando Mascarenhas (2005) tiene en su tesis de doctorado, la matriz de aquello que se está materializando en textos publicados en un conjunto de obras y periódicos del área. Su comprensión conceptual de tiempo libre está incorporada al documento Política Nacional de Deporte, habiendo servido de referencia para el proyecto social Deporte y Tiempo Libre de la Ciudad en el primero mandato del gobierno Lula.

9 La disertación de maestría de Luciana Marcassa (2002) es referencia obligatoria para los que desean comprender el proceso de institucionalización del tiempo libre en Brasil. También se hace pertinente la visita a la tesis de doctorado de Valquíria Padilha (2003).

10 EI Ministerio de la Cultura, en alianza con el IBGE, elaboró diagnóstico que apunta no haber diferencia porcentual de comprometimiento de sus presupuestos mensuales entre la población de baja renta y los más ricos (4\%). Informa también que $87 \%$ de los brasileños nunca fueron al cine y $92 \%$ a los museos; $78 \%$ nunca vieron espectáculos de danza. 
mismo tiempo que los indicadores de calidad de vida revelan el hecho de que el Brasil continúa situado entre los de mayor desigualdad social, señalizador de que las políticas sociales implementadas en estos últimos años si, por un lado, registran mejorías en la alteración del carácter concentrador de renta de nuestro modelo económico, por otro lado, aún se muestran insuficientes para alterar el desalentador escenario.

Así, datos relativos al (i) ingreso de Brasil en el grupo de los países con más alto índice de desarrollo humano ${ }^{11}(\mathrm{IDH})$, sumados a las noticias sobre (ii) el mejor resultado en 11 años de la renta del trabajador (7,2 \% en 2006), asociado a la menor tasa de desempleo desde 1997, (iii) la disminución del trabajo infantil, (iv) la mayor accesibilidad a los bienes y servicios por parte de la población, como también a la escolarización (97,6 \% están en la escuela y la enseñanza superior tuvo un crecimiento de 13,2 \%), conviven con (i) la incapacidad de su mayor programa social (Asignación Familiar [Bolsa Família]) de vencer la miseria en siete Estados y en la región noreste, conforme demuestra un estudio elaborado por el propio Ministerio del Desarrollo Social, (ii) el aún enorme contingente de trabajadores en la economía informal y, peor, los que están en condiciones caracterizadas como de trabajo esclavo, con (iii) 5,1 millones de niños todavía en situación de trabajo infantil y con (iv) una educación básica y superior vergonzosamente descalificadas.

Es lo que extraemos en el análisis de los datos más recientes de la Encuesta Nacional por Muestreo de Domicilios (Pnad) ${ }^{12}$ divulgada anualmente por el Instituto Brasileño de Geografía y Estadística (IBGE) y del índice de Gini ${ }^{13}$, que cayó de 0,544 para 0,541 en

11 Ya en 1996 me valí de este raciocinio en un artículo denominado Tiempo libre y Calidad de Vida publicado en un conjunto de obras titulas de Políticas Públicas Sectoriales de Tiempo libre, editada por Autores Asociados. En él me apoyo en el Informe/95 del Programa de las Naciones Unidas para el Desarrollo acerca del Índice de Desarrollo Humano, como también me reporto a la HABITAT 2, Conferencia Mundial sobre el proceso de urbanización en el Mundo, organizada por el Centro de las Naciones Unidas para los Asentamientos Humanos de la Organización de las Naciones Unidas, ONU, realizada en el período de 3 a14 de junio de 1996 en Estambul, Turquía.

12 EI Pnad/IBGE -la más amplia investigación sobre la realidad del país- verifica las características domiciliares en relación al acceso a bienes y servicios, al alcance a la educación, al panorama del mercado de trabajo ya la evolución del rendimiento del trabajador.

13El índice de Gini es una de las formas de medir el proceso de distribución de renta del trabajo (excluidas las transferencias
2006, en una serie histórica de naturaleza lenta y regular iniciada hace poco más de una década y reveladora de la presencia de Brasil entre los países más desiguales, donde el $10 \%$ más pobre entre los trabajadores se quedó con $1 \%$ de los rendimientos en 2006, mientras que el $10 \%$ más rico obtuvo $44,4 \%$.

\section{DEPORTE Y TIEMPO LIBRE BAJO LA PERSPECTIVA DE LA EDUCACIÓN FÍSICA ${ }^{14}$}

Deporte y tiempo libre se constituyen por conceptos distintos, en los cuales el último no puede ser reducido a las fronteras del primero. En ese sentido, vale decir que el deporte recreativo -también deporte de tiempo libre y deporte de participación- exige una política específica que lo haga incorporarse a una política de tiempo libre, también especifica que no se limite a desarrollarse, única y exclusivamente, por intermedio del interés físico deportivo. Al contrario, es fundamental abrirse a la inevitable necesidad de contemplar la inmensa gama de intereses que se presentan en nuestro cuadro cultural.

Los motivos que llevaron a la educación física a colocarse como protagonista de las acciones en el campo del tiempo libre, asociadas al hecho de ser pensado en el mundo del deporte, casi que resumiéndose a él ${ }^{15}$, viene de otros momentos y situaciones anteriores a aquel donde el deporte aparece como producto de la industria cultural del entretenimiento.

A continuación se enumeran, entonces, los pasos constitutivos de esta relación de sumisión del tiempo libre a la óptica del deporte ${ }^{16}$ :

como el Bolsa-Família (Asignación Familiar) y las jubilaciones). La distribución varía de 0 a 1, indicando mayor desigualdad a medida que aumenta.

14 Educación física, aquí, es asumida como área académica tal cual es tratada por agencias gubernamentales de fomento a la investigación (CNPq, Fapesp...) como también por la Capes.

15 El hecho del tiempo libre es tratado en la Carta Magna brasileña de 1988 en el capítulo del "Deporte", por un lado refleja la construcción histórica de esta relación y, por otro, la refuerza.

16 Las reflexiones siguientes son fragmentos de la primera versión del texto básico de la I Conferencia Nacional del Deporte (junio/2004), elaborada por mí en la condición de Secretario Nacional de la Secretaría Nacional de Desarrollo del Deporte y del Tiempo Libre, órgano del Ministerio del Deporte. Otras dos versiones se crearon hasta la definición de aquella llevada al conocimiento de los delegados participantes de dicha Conferencia. 
- La presencia del Estado brasileño en los asuntos del deporte es incontestable, demostrada en la propia historia de la organización social y política del país, en especial aquella construida a partir de la segunda década del siglo pasado, cuando Brasil dejaba atrás el modelo económico agrario de naturaleza comercial -exportadory se organizaba dentro del molde de la industrialización, pautada por el modo de producción capitalista.

- Es, sin duda, la inserción de Brasil en el modelo económico industrial, la responsable por el proceso de urbanización vivido por la sociedad brasileña a lo largo del siglo XX. A principios de aquel siglo, 9,40 \% de la población vivía en ciudades; y a mediados de los años 1940 era 31,24 $\%$, llegando a expresivos $81,23 \%$ en su última década.

- Las ciudades, a su vez, pasaron a exigir de los gobernantes la construcción de políticas públicas que respondieran a los enormes desafíos de los cada vez mayores aglomerados urbanos. Entre ellas, no tardaron en surgir iniciativas gubernamentales dirigidas al mantenimiento del orden público, donde preocupaciones por la recreación obrera y de los otros habitantes de la ciudad se materializaron incorporando, en su interior, las prácticas corporales y deportivas como intereses socioculturales predominantes.

- No demoró mucho para que el deporte pasara a merecer la atención especial del Gobierno federal de aquella época. Con la llegada y las características dictatoriales propias del periodo del Estado Novo, fue objeto de la interferencia normativa del Estado. Tal interferencia, allí iniciada, puede ser demostrada a partir de dos conjuntos de documentos legales, explicativos de la pretensión estatal de definir directrices y bases para el deporte nacional.

- El primero de ellos se remonta a la década de 1940, cuando por primera vez el Gobierno brasileño, impulsado por la lógica del Estado Nuevo, buscó -en expresión presente en la Exposición de Motivos del Decreto Ley 3.199/41- disciplinar al deporte brasileño.
- Tal procedimiento acabó representando una línea intervencionista de naturaleza conservadora, gracias a su carácter proteccionista, propio al perfil estadonovista que vigoró de 1937 a 1945.

- Sumado a esto estaban presentes intereses económicos y políticos. El primero de ellos estaba asociado a la intención de perfeccionamiento de la aptitud física de la población, con el objetivo de la capacitación física del trabajador, como expresaba la Constitución brasileña de 1937.

- Ya los intereses políticos mantenían estrecha relación con el deseo de desarrollar del espíritu cívico y patriótico de tono afinado con la posición brasileña frente a la coyuntura internacional.

- Esta intervención se sedimentó a lo largo de los 34 años en que tal Decreto Ley rigió la vida deportiva brasileña, con lo cual se consolidó en otro momento de régimen de excepción, a través de la Ley 6.251 de 1975, reglamentada, dos años más tarde, por el Decreto 80.228.

- Esta Ley se caracterizó por traer alteraciones superficiales a las normas deportivas hasta aquel momento existentes, manteniendo básicamente las mismas directrices y principios orientadores del documento de 1941, dando flujo también en este sector, de lo que en otros se llamó de modernización conservadora.

- Al establecer los objetivos de la Política Nacional de Educación Física y Deporte, enumerados en su artículo 5, mostraba haber sido la aptitud física la hipótesis básica sobre la cual se estaba asentando el entendimiento dominante para la construcción de la política pública para este sector. En efecto, a partir del paradigma de la aptitud física se explica la visión a través de la cual la educación física y el deporte estaban siendo tratados por las políticas del área.

- Siendo de esta forma, los incisos II (elevación del nivel de los deportes en todas las áreas) y IV (elevación del nivel técnico deportivo de las representaciones nacionales) tenían sentido si -al afinarse con el concepto de deporte enunciado en el artículo 2 de la ley en pauta (para los efectos de esta ley se considera deporte a la actividad 
predominantemente física, con finalidad competitiva, ejercitada conforme reglas preestablecidas)-fueran analizados en la dirección de reforzar la posibilidad de alcanzar el objetivo enunciado en el inciso I del mismo artículo (perfeccionamiento de la aptitud física de la población).

- Lo mismo se puede afirmar de los incisos III (implantación e intensificación de la práctica de los deportes de masa) y $\mathbf{V}$ (difusión de los deportes como forma de utilización del tempo libre ${ }^{17}$. Tales incisos se inscribieron en el ámbito de las respuestas a las preocupaciones de la clase dominante, de tratar de demostrar que al pregonado desarrollo económico alcanzado en aquella primera mitad de la década de 1970 época del milagro brasileño-, correspondía un equivalente desarrollo social que, en el campo de la educación física y del deporte, llevaba la posibilidad de acceso a las actividades físicas de tiempo libre al sector de la población hasta entonces de ellas descartadas.

- Así, al limitar la educación física y el deporte respectivamente a sus dimensiones de práctica corporal y deportiva, se inscribían también, tales incisos, en los esfuerzos de atender al precepto en el ya aquí mencionado inciso I (perfeccionamiento de la aptitud física de la población), a su vez sustentado por un referencial de salud de índole biofisiológica.

- De esta manera, educación física y deporte fueron confundidos con educación del físico, educación del cuerpo, de su rendimiento físico deportivo, simulacros acerca de la productividad, eficiencia y eficacia inherente al modelo de sociedad en el cual la brasileña encontraba identificación.

\section{ABRIENDO UN PARÉNTESIS PARA LA FORMACIÓN PROFESIONAL}

Cabe aquí un breve paréntesis para que tratemos del tema de la formación de profesionales que asumieran la tarea de continuar la ejecución de las políticas

17 Subrayado nuestro. habituadas a la lógica de la recreación pública como también del deporte, regularizado dos años después.

Es de 1939 la creación en Río de Janeiro, capital del país en aquel momento, de la Escuela Nacional de Educación Física junto a la Universidad de Brasil (hoy UFRJ).

El Decreto Ley 1212/39, al crear aquel curso universitario, estableció los parámetros curriculares a partir de los cuales el cuerpo docente -constituido básicamente por médicos y militares- daría flujo al proceso de formación profesional, atentos a las exigencias establecidas por la lógica del Gobierno estadonovista.

Solamente en 1969 se observa una reforma curricular de los cursos universitarios de educación física entonces existentes, de esta acción realizada bajo los auspicios del Consejo Federal de Educación y expresada a través del Resolución 69.

Así, se observa el refuerzo de la lógica del documento anterior, apenas ahora sintonizada con la Doctrina de Seguridad Nacional en vigor bajo la tutela de los gobiernos militares que se sucedían desde 1964. Productividad, eficiencia y eficacia, combinadas con la sedimentación del proceso de urbanización de la sociedad brasileña y la defensa de la patria de los que discordaban del Gobierno de excepción, daban al proceso de formación profesional un sello técnico instrumental, excluyendo más que dejando en segundo plano- la estructuración de una sólida base teórica de cuño humanístico que los habilitaran a darse cuenta del tiempo en que vivían, lo que favoreció la formación de profesionales acríticos, en detrimento a la de gestores con conocimiento de los determinantes del ordenamiento societario brasileño, su naturaleza excluyente y de los procesos de planificación en deporte y tiempo libre, razonables para la reversión del cuadro de exclusión de la mayoría de la población a su acceso.

Asociada a las perspectivas ya mencionadas, la formación de profesionales para el área de tiempo libre está tanto ampliándose, cuantitativa y cualitativamente, como diversificándose ${ }^{18}$. Tanto buscando adecuarse a la demanda del mercado, de forma de someterse a

\footnotetext{
18 Cursos superiores de turismo se suman a los de tiempo libre y de educación física en la formación de profesionales para el tiempo libre, dando muestras del carácter multidisciplinar del proceso de formación y del sentido transversal e intersectorial inherente a las políticas asignadas al área.
} 
él, como de establecer una relación dialéctica con el mundo del trabajo, centrada en una formación profesional al mismo tiempo calificadora de la inserción en el universo de trabajo y celosa de las relaciones en él existentes, abriéndose a la posibilidad de transformarlas ${ }^{19}$.

Cerremos el paréntesis y volvamos a la lógica anterior:

- Entonces el conjunto legal hasta aquí referido -reforzador de la oligarquización de la organización deportiva- fue sustituido por otro, motivado por la promulgación de la Constitución brasileña de 05 de octubre de 1988 y formateado bajo la fuerte influencia de los referenciales neoliberales de Estado y Gobierno que pasaron a regir en nuestro medio.

- Constituido por la Ley Zico (Ley 8.672/93 y Decreto 981/93) y por la Ley Pelé (Ley 9.615/98 y Decreto 2.574/98), este otro conjunto dio lugar a lo descrito por Meily Assbú Linhales ${ }^{20}$ como un proceso de cambios dirigido fundamentalmente por el confronto entre los intereses liberalizadores -que buscaban autonomía de mercado para el deporte- y los intereses conservadores, que entendían esa liberalización como una amenaza al poder constituido oligárquicamente.

- El embate entre liberalizadores y conservadores fue responsable por el surgimiento de la autonomía y de la pluralidad como elementos de cambios. Contradictoriamente, se mezclaban a los trazos dejados por la conservación de antiguas prácticas. Tal línea de reflexión -aún de acuerdo

19 El Bachiller en Tiempo Libre ofrecido por la Facultad de Educación Física de la Unicamp hasta 2005 y el área de concentración Educación Física y Sociedad de su programa de posgrado, se encuadraban entre aquellos preocupados con privilegiar la formación crítica en detrimento de la técnico instrumental. En la misma dirección tenemos, a partir de 2007, la maestría en Tiempo Libre de la Universidad Federal de Minas Gerais -y a partir de 2011, el doctorado-, ofrecido en el ámbito de su Facultad de Educación Física, Fisioterapia y Terapia Ocupacional. Vale destacar la estructuración en el ámbito de la Facultad de Educación Física de la UNB - Universidad Federal de Brasília -del Grupo de Estudios e Investigaciones "Avante" que, bajo la coordinación de los profesores Edson Marcelo Hungaro y Fernando Mascarenhas fortalecen el campo de la teoría crítica a partir del referencial materialista-histórico-dialéctico.

20 El pensamiento de la profesora Meily aquí apropiado deriva de su disertación de maestría ("La trayectoria política del deporte en Brasil: intereses involucrados, sectores excluidos") defendida en 1996 junto al área de ciencias políticas del Programa de Posgrado de la Facultad de Filosofía y Ciencias Humanas de la UFMG. con Meily- nos condujo a un cuadro donde, en ninguna de las dos direcciones, se consolidaron alternativas que vieran al deporte como una necesidad o derecho social, y tampoco al Estado como mediador y punto de equilibrio entre los intereses antagónicos existentes en el sector deportivo.

\section{Y AQUÍ, LA ESPERANZA AÚN NO VENCIÓ AL MIEDO...}

Con la llegada del PT al Gobierno Federal, aunque limitada por las alianzas al centro y de él, a la derecha, señalizó para la posibilidad del hasta entonces contrahegemónico rastrear la indicación de parámetros para que la democratización del deporte (y de la liberación del tiempo libre de su yugo) pudiera ocurrir y ser usufructuada por todo el conjunto de la población brasileña.

Se derivó de ahí la necesidad de establecer las condiciones para que se construyera una concepción del espacio de la vivencia de las prácticas corporales y deportivas como lugar privilegiado de elevación del nivel de su consideración, enriqueciéndolas de valores que interactuasen en la construcción de una ciudadanía plena.

En este entendimiento se sustentaría el principio de la inclusión: primeramente, entendiendo ser deber del Estado garantizar a toda la sociedad el acceso al deporte y al tiempo libre, identificando en ellos la capacidad privilegiada de pasar a contribuir, al lado de otras acciones de gobierno, en los esfuerzos de inclusión social (derivándose entonces el eslogan inclusión social a través del deporte) de un grupo significativo de la población brasileña.

Se materializaría de esta forma, el objetivo de señalizar para la inversión de la lógica de la presencia del Estado en el campo deportivo, atribuyéndole prioritariamente un carácter de subsidio; sin embargo, de otro sentido que no aquel reforzador del modelo concentrador, representado gráficamente por una pirámide trayendo, en su vértice, el deporte de alto rendimiento $y$, en su base y centro, respectivamente, el deporte de masas y el deporte estudiantil subordinados a los objetivos del de arriba.

Lo que se deseaba proponer era la implementación de un modelo ejemplificado por círculos autónomos $\mathrm{y}$, al mismo tiempo, interdependientes, en los cuales 
el deporte recreativo, el estudiantil y el de alto rendimiento fueran respetados en sus especificidades y, en un mismo momento, mantuvieran canales de comunicación señalizadores de un concepto de sistema deportivo construido en relaciones isonómicas y no jerarquizadas, respetándose para la financiación pública de esas dimensiones lo establecido en la Constitución brasileña.

En paralelo, relajándose el cuadro, se visualizaría el tiempo libre en toda su plenitud, cada vez más libre de las amarras de la cultura del entretenimiento y no más sometido a la dominación de la institución deportiva, cada vez más propenso a ser asumido como política de estado de índole transversal e intersectorial.

Pues la esperanza traducida en posibilidad histórica no se tradujo en realidad histórica, frustrando la expectativa de muchos y empujando hacia adelante la materialización de lo que aún permanece en el universo de la utopía (Castellani, 2005) ${ }^{21}$.

\section{DEPORTE, TIEMPO LIBRE Y ESCUELA}

Habiendo hablado del deporte, del tiempo libre y de nuestra comprensión del concepto de inclusión social, cabe a partir de ahora, elaborar consideraciones sobre la relación deporte, tiempo libre y escuela, no sin antes decir cómo vemos a esta última.

Para nosotros la escuela es lugar de formación humana realizada a partir del desarrollo del proceso de conocimiento; lugar de aprehensión de la realidad; aprehender configurado en sus expresiones de constatar, demostrar, comprender y explicar la realidad compleja en la cual nos encontramos insertados.

Sin embargo, lejos de tener la aprehensión de la realidad como punto de llegada del proceso de formación,

21 La II Conferencia Nacional de Deporte (mayo/06) se realizó bajo el tema "Construyendo el Sistema Nacional de Deporte y Tiempo Libre". La inclusión del Tiempo Libre en el Sistema fue posición extraída en un Fórum organizado por el Ministerio del Deporte en octubre de 2005. Tal posición molesto a sectores vinculados al campo deportivo (como también a otro en el propio ministerio, próximo al gabinete del ministro y dispuesto a no herir la sensibilidad de los dirigentes deportivos), que subestimó el fórum, a él compareciendo en menor número que los defensores de la inclusión del tiempo libre. En la II Conferencia, "limpiada el área" intra ministerio defensora de la inclusión, prevaleció la posición de la exclusión del Tiempo Libre en la configuración del Sistema, apenas siendo incorporado el deporte de participación como referencia a él, Tiempo Libre. la vemos como punto de partida para el movimiento de intervención en esta misma realidad, a efectuarse de forma autónoma, crítica y creativa.

El proceso pedagógico en ella instalado está dotado de sentido diagnóstico (pues parte de la comprensión de la realidad establecida como concreto real transmutándola por la teorización en concreto pensado), sentencioso (pues establece juicio de valor demarcado por el origen, posición y situación de clase social de los involucrados en el proceso enseñanza/aprendizaje) y teleológico (pues señaliza un objetivo a alcanzar, un determinado lugar a ser ocupado por los sujetos sociales).

Así, entendemos ser responsabilidad de la educación física -componente curricular de la educación básicael tratamiento pedagógico de los temas constitutivos de la cultura corporal.

A través de ella se comprende que el deporte deba hacerse presente en el ambiente escolar. No obstante, esto por sí solo no es garantía de que venga a merecer un tratamiento pedagógico reconocedor de su condición de parte integrante de la cultura de un pueblo. Esto porque, en el caso brasileño, la relación paradigmática de la educación física con el concepto de aptitud física hizo reforzar su subordinación a las instituciones médica y militar y a la consecución de su relación con la institución deportiva pautada casi que exclusivamente por el principio del rendimiento físico deportivo.

En verdad, la relación paradigmática ya citada se extendió hegemónicamente de mediados del siglo XIX por casi todo el siglo siguiente, proceso durante el cueal se encontraron resistencias de orden contrahegemónico solamente a partir de la década de 1980, la cual dio origen a una relación paradigmática de cuño históricos social, a partir de la cual el deporte pasó a ser valorado su sentido de práctica social, expresión de nuestra cultura.

Estudios sobre la presencia del deporte en la escuela, interrogadores de la relación entonces dominantemente presente, ganaron impulso a partir del fin de la década de 1970, comienzos de la de 1980. Y no es casualidad, pues como es sabido, dicho periodo coincide con el de la redemocratización de la sociedad brasileña responsable por el proceso de oxigenación 
de las instituciones, dotándolas de aires renovados y repletos de la voluntad política de construcción de un nuevo tiempo.

Recientemente se realizaron nuevas incursiones sobre la problemática, con el propósito de sedimentar el camino para nuevas posibilidades de análisis de la presencia del deporte en el escenario educacional. Entre ellas, se destaca la realizada por Sávio Assis de Oliveira, ya publicada en el libro denominado "Reinventando el deporte: posibilidades de la práctica pedagógica”.

El mismo autor, en su proyecto de estudio de doctorado (“¿Deporte y escuela en el mismo equipo? Evaluando las políticas públicas de deporte escolar en el Brasil del siglo XXI"), al tiempo que examina consideraciones sobre el asunto, se reporta a otras iniciativas, en marcha, de estudio de la temática, como el de la profesora Meily Assbú Linhales, también asociado a estudios de doctorado, denominado "La escolarización del deporte: un estudio sobre la 'llegada' del deporte a la escuela brasileña, 1930-1940".

Tarcísio Mauro Vago, Elenor Kunz, Valter Bracht, Mauro Betti, Marcos Taborda son, entre otros, investigadores que también se interesaron sobre la relación deporte y escuela.

La responsabilidad de la educación física escolar por la educación deportiva entendida a partir del parámetro del deporte de alto rendimiento, además de enmascarar el quiebre del modelo de iniciación deportiva centrada en los clubes sociales - "célula mater" del deporte brasileño, como se acostumbraba a decir antiguamente-, inhibió el prevalecer de concepciones pedagógicas no apoyadas en la lógica de referencia, al mismo tiempo en que fortaleció iniciativas, presentes en los campos del ejecutivo y legislativo, ratificadoras del arquetipo conceptual sustentador de aquel modelo.

En el campo legislativo federal tuvimos, en 1994 (05/12/94), la aprobación de la Ley 8.946 (después revocada por la Ley Pelé) creando el Sistema Educacional Deportivo integrado al sistema brasileño de deporte. En 2004 se tramitó en la Cámara Federal el Proyecto de Ley 626/03 que proponía, entre otras cosas, la educación deportiva como componente curricular obligatorio desvinculada de la educación física, esta última enseñada por profesores y aquella también por profesores de deporte reclutados entre los deportistas de alto nivel que ingresarían al magisterio público, conforme criterios propuestos en los párrafos 1, 2 y 3 del artículo 6, los cuales los dispensaban de la obligatoriedad de pruebas de títulos y documentos, exceptuada la obligatoriedad de comprobación de conclusión de la educación básica.

En el campo del ejecutivo federal, el Programa Deporte en la Escuela fue lanzado en 2001 por el Gobierno FHC anunciado como uno de los más importantes del área social de su gobierno. En 2003, ya en el primer mandato del Gobierno LULA, fue presentado a la sociedad, también con derecho a ceremonia en el Palacio del Planalto, el Programa Segundo Tiempo. Ambos son susceptibles de análisis detallados ${ }^{22}$.

En el Estado de São Paulo, en periodo equivalente, tuvimos el Aliados del Futuro (ya analizado en disertación de maestría defendida junto al Programa de Posgrado en Educación de la USP, en 2004) ${ }^{23}$ sustituido por el Programa Escuela de la Familia.

Por todo lo anterior, se puede afirmar que la relación deporte/escuela está lejos de caracterizarse como tranquila, exenta de tensiones motivadas, la mayoría de ellas, por la dificultad en la compatibilización de los objetivos de una y de otra institución (la escolar y la deportiva).

En algunos momentos, el deporte entró en la escuela sin pedir permiso, obligándola a aceptar sus códigos y significados; en otros fue recibido de brazos abiertos, a medida que se proponía llevar su nombre a los más altos, y gran mayoría de veces, inimaginables lugares (la asimilación bastante rápida por parte de la escuela del bajo costo del marketing conseguido a través de él tiene una parte considerable de responsabilidad por esta postura amistosa); en otras tantas la lógica instrumental fue la responsable por la autorización de su ingreso.

Solamente en menores ocasiones deporte y escuela compartieron sentimientos: él, notándola como

\footnotetext{
22 A quien desea enfrentar el desafío, vale la pena explorar la manifestación de alivio y alegría de técnicos del ministerio de planificación cuando se enteraron del Programa Segundo Tiempo y notaron el hecho del mismo obedecer a la misma concepción presente en el Programa del Gobierno anterior, Deporte en la Escuela. Conforme ellos, ¡la "serie histórica" estaba preservada!

23 "La escuela pública y el tiempo libre: un estudio de caso del Programa Aliados del Futuro" es el título de la disertación defendida por Reinaldo Tadeu B. Pacheco, que la elaboró bajo orientación del profesor Vitor Paro.
} 
espacio de formación humana y no como lugar estratégico de detección de talentos deportivos; ella, reconociendo la existencia de conocimiento en el deporte además de aquel situado en el campo del saber hacer deportivo, sino también en el universo de los significados que posee en el ámbito de nuestra cultura y de su importancia en la construcción de nuestra existencia.

De esta forma, en la relación deporte/escuela, ambos son a la vez aprendices y profesores. También aquí vale la pena resaltar que en el proceso enseñanza/aprendizaje todos tienen algo que aprender y enseñar. El deporte no estará de visita en la escuela en el instante en que deje de identificar a la escuela como un lugar más para hacer valer los propósitos del sistema deportivo; la escuela lo tratará como parte constitutiva del proceso de escolarización cuando reconozca en él un atributo de emancipación humana.

Cuando esto ocurra, el tiempo libre se hará presente en la escuela no como espacio de recreación de los alumnos, ni sometido a la institución deportiva, sino como contenido programático de proyectos de escolarización atentos a la importancia de la educación lúdica en el proceso de formación humana de los que por ella pasen.

Defendemos la presencia del tiempo libre en la educación básica en nuestro país a partir de múltiples entradas, aunque observemos que el componente curricular educación física está asumiendo la primacía -y responsabilidad- de dar tratamiento al tema en el ámbito del proceso de escolarización.

En su ámbito, concepciones pedagógicas que permitan tal acción ya existen (como la denominada críticosuperadora), con un más que razonable grado de elaboración teórica y sistematización metodológica para su enseñanza. No defendemos, sin embargo, la prerrogativa de la exclusividad del tratamiento a ser dado al tema tiempo libre, en el universo de la educación básica, a ella, la educación física ${ }^{24}$. Lo fundamental es que los procedimientos de enseñanza favorezcan la educación lúdica -suposición de la cultura lúdica- del educando, teniendo como parámetro los elementos constitutivos de la óptica contra hegemónica anteriormente aludida.

24 Incluso porque defendemos la superación de la estructura disciplinar en la configuración de la dinámica curricular escolar, haciendo posible esta presencia en la Ley de Directrices y Bases de la Educación Nacional en vigor.

\section{EN EL CAMINO DE LAS INVESTIGACIONES EN DEPORTE Y TIEMPO LIBRE...}

A nosotros nos resta continuar construyendo las condiciones objetivas para la concretización de nuestras utopías.

En lo pertinente a los desafíos presentes a los que poseen en el deporte y en el tiempo libre, sus objetos de investigación, se observa la necesidad de la ampliación de la tendencia de la asociación de los estudios a aquellos que hoy reflejan el actual nivel de desarrollo del modo de producción capitalista que, bajo el nombre genérico de globalización, está construyendo un mundo pautado en la exclusión social que reserva a cada vez menos segmentos sociales el acceso a la riqueza material y cultural producida por la humanidad a lo largo de su proceso civilizatorio.

Es en este terreno que percibimos pertinente los estudios acerca de las teorías del deporte y del tiempo libre, de los procesos de formulación de políticas, y de la planificación y gestión del deporte y del tiempo libre, de la educación lúdica y deportiva y, para que no nos extendamos, de la formación profesional/académica de los que van a inmiscuirse en los asuntos relativos al área del tiempo libre y del deporte.

La calidad de los estudios e investigaciones desarrolladas por grupos vinculados a nuestras universidades ${ }^{25}$ abre amplia y positiva expectativa en relación al futuro. El desafío está en hacer que estos grupos sean cada vez más de frente para la realidad de las políticas de educación, deporte y tiempo libre en los más de 5560 municipios brasileños, en sus estados y en el propio Gobierno central, sean ellas públicas o privadas ${ }^{26}$.

A su vez, el descrédito en la clase política-motivado por la degradación de la estructura partidaria, cuya desideologización programática hace prevalecer la búsqueda del poder concentrado y responsable por gran parte de los escándalos denunciados (y de los aún

25 El directorio de Grupos del CNPq trae el registro de los grupos existentes y de sus producciones. Sin desmerecer a los demás, resalto los de UFRGS, UFMG, UFPR, UFG, UFU, UFRJ, UFES, UFBA, UFMA, UNB, CESET/RN, IMES/SCS, USP, UNESP y Unicamp.

26 Fue con el objetivo de atender las demandas de la gestión pública en deporte y tiempo libre, por estudios e investigaciones que calificaran sus acciones, que el proyecto de la RED CEDES fue elaborado en el interior del Programa presupuestario Deporte y Tiempo Libre de la Ciudad, manteniendo en el periodo 2004/07 coherencia con el principio en pauta 
cubiertos)- necesita ser visto en aquello que señaliza para la necesidad de la madurez de nuestra democracia, señalada en el fortalecimiento de sus instituciones y en la capacidad política de la sociedad civil de establecer control social de las acciones gubernamentales y no gubernamentales.

\section{REFERENCIAS BIBLIOGRÁFICAS}

Assis de Oliveira, S. (2001). La reinvención del deporte: posibilidades de la práctica pedagógica. Campinas: Autores Asociados.

Bracht, V. (1997). Sociología crítica del deporte: Una introducción. Victoria: UFES, Centro de Educación Física y Deportes.

Congreso Nacional (1988). Constitución de la República Federativa de Brasil de 05 de octubre de 1988. Brasilia.

Gobierno Federal (2004). Programa Deporte y Tiempo Libre de la Ciudad. Brasilia. "Plano Plurianual de Gobierno: Brasil un país de Todos".

Ministerio del Deporte (2004). Conferencia Nacional de Deporte - Documento Final. Brasilia: Gobierno Federal.

Ministerio del Deporte (2005). Programa Deporte y Tiempo Libre de la Ciudad - Manual de Orientación. Brasilia: Gobierno Federal.

Ministerio del Deporte (2006). Política Nacional de Deporte. Brasilia: Gobierno Federal.

Ministerio del Deporte - IBGE (2006). Suplemento de Deporte y Tiempo Libre - Encuesta Perfil de los municipios brasileños. Río de Janeiro: Gobierno Federal.

Ministerio del Deporte (2006) II Conferencia Nacional de Deporte. Brasilia: Gobierno Federal.

IBGE (2007). Encuesta Nacional por Muestreo de Domicilios (Pnad). Río de Janeiro: Gobierno Federal.

Ministerio de la Cultura - IBGE. (2007) Suplemento Cultura - Encuesta Nacional por Muestreo de Domicilios (Pnad). Río de Janeiro: Gobierno Federal.

Castelan, L. (2011). Las Conferencias Nacionales del Deporte en la configuración de la Política Deportiva y de Tiempo Libre en el Gobierno LULA (2003-2010). (Disertación inédita de Maestría). Unicamp, Campinas, SP.

Castellani Filho, L. y Carvalho, Y. M. (2006). Resignificando el Deporte y el Tiempo Libre en las relaciones con la Salud. En: A. Castro y M. Malo (org.). SUS: Resignificando la Promoción de la Salud (pp. 208-222). São Paulo: Hucitec.
Como ya cantaron, "aprendemos mucho en ese tiempo”... Entonces, “¡desesperar, jamás! Al final de cuentas, ¡no tiene cabida entregar el partido en el primer tiempo!...".

Castellani Filho, L. (1996). Tiempo libre y calidad de vida. En: N.C. Marcellino (org.). Políticas públicas sectoriales de tiempo libre (pp. 7-21). Campinas: Autores Asociados.

.(1999) La Educación Física en el Sistema Educacional Brasileño: Caminos, paradojos y perspectivas. (Tesis inédita de Doctorado). Unicamp, Campinas, SP.

(2001). Notas para una agenda del deporte brasileño. En Colección de textos de la 1a Conferencia Nacional de Educación, Cultura y Deporte. Desafíos para el Siglo XXI (pp. 577-589). Brasilia: Cámara de los Diputados. Coordinación de Publicaciones. Serie Acción Parlamentar no 146. . (2002). Política Educacional y Educación Física. Campinas: Autores Asociados.

(2004). Deporte y Tiempo Libre de la Ciudad. En: C. Turino (coord.). Recreo en las vacaciones: una experiencia de política pública de tiempo libre y educación (pp. 69-80). São Paulo: IMK Relaciones Pública.

- (2005). Las prácticas corporales y su proceso de resignificación. En: I.R. Damiani y A.M. Silva (org.). Prácticas Corporales: Génesis de un Movimiento Investigativo en Educación Física (pp.1115). Florianópolis: Nauemblu Ciencia y Arte.

(2006). Gestión Municipal y Política de Tiempo Libre. En: M. Linhales y H. Isayama (org.). Sobre tiempo libre y política: maneras de ver, maneras de hacer (pp. 121-135). Belo Horizonte: UFMG.

Colectivo de autores. (2012). Metodología de la Enseñanza de Educación Física. São Paulo: Editora Cortez.

García Canclini, N. (1983). Políticas culturales en América Latina. Nuevos Estudios 2(2), 39-51.

Global Entertainment and Media Outlook: 2007-2011 (s.f.). En: $P W C$. Recuperado: http://www.pwc.com/ id/en/publications/entertainment-media-globaloutlook-2007-2011.jhtml 
Gonçalves, M.A. (1997). Intercambio aproxima países y anuncia "cultura global". Folha de São Paulo, Suplemento Especial - Globalización, 02/11/1997, p.10.

Hobsbawn, E. (1997). Era de los extremos: el breve siglo XX. 1914-1991. São Paulo: Compañía de las Letras.

Ianni, O. (1997). La sociedad global. Río de Janeiro: Civilización Brasileña.

Linhales, M. A. (1996). La trayectoria política del deporte en Brasil: intereses involucrados, sectores excluidos. (Disertación inédita de Maestría). UFMG, Belo Horizonte, Minas Gerais.

Marcassa, L. (2002). La invención del Tiempo Libre: Educación, Cultura y Tiempo Libre en la ciudad de São Paulo (1888-1935). Disertación inédita de maestría. Goiânia, Goiás: Universidad Federal de Goiás.
Mascarenhas, F. (2005). Entre el ocio y el negocio: tesis acerca de la anatomía del tiempo libre. Tesis inédita de doctorado. Campinas: Unicamp.

Nascimento Neto, A. (1996, 3 de abril). La Rueda Global. Revista Veja(14), 80-89.

Padilha, V. (2003). Shopping Center: La catedral de las mercancías y del tiempo libre transformado. (Tesis inédita de Doctorado). Unicamp, Campinas, SP.

Pachego, R.T.B. (2004). La Escuela Pública y el Tiempo Libre: Un estudio de caso del Programa "Aliados del Futuro". (Disertación inédita de Maestría). USP, São Paulo.

Toledo, R. P. (1996, 3 de abril). La Fuerza de la Aldea. Revista Veja, (1438), 92-93. 